\title{
Augmenting Sparse Laser Scans with Virtual Scans to Improve the Performance of Alignment Algorithms
}

\author{
Rolf Lakaemper \\ Department of Computer and Information Science Temple University, Philadelphia, USA \\ lakamper@temple.edu
}

\begin{abstract}
We present a system to increase the performance of feature correspondence based alignment algorithms for laser scan data. Alignment approaches for robot mapping, like ICP or FFS, perform successfully only under the condition of sufficient feature overlap between single scans. This condition is often not met, e.g. in sparsely scanned environments or disaster areas for search and rescue robot tasks. Assuming mid level world knowledge (in the presented case the weak presence of noisy, roughly linear or rectangular-like objects) our system augments the sensor data with hypotheses ('Virtual Scans') about ideal models of these objects, based on analysis of a current estimated map of the underlying iterative alignment algorithm. Feedback between the data alignment and the data analysis confirms, modifies, or discards the Virtual Scan data in each iteration. Experiments with a simulated scenario and real world data from a rescue robot scenario show the applicability and advantages of the approach.
\end{abstract}

\section{Introduction}

Robot mapping based on laser range scans is a major field of research in robotics in the recent years. The basic task of mapping is to combine spatial data usually gained from laser range devices, called 'scans', to a single data set, the 'global map'. The global map represents the environment scanned from different locations, even possibly scanned by different robots ('multi robot mapping'), usually without knowledge of their pose (= position and heading). One class of approaches to tackle this problem, i.e. to align single scans, is based on feature correspondences between the single scans to find optimal correspondence configurations. Techniques like ICP (Iterative Closest Point, e.g. [2, 24] and [22]) or FFS (Force Field Simulation based alignment, [20]) belong to this class. They show impressive results, but are naturally restricted: first since they are feature correspondence based, they require the presence of a sufficient amount of common, overlapping features in scans belonging together. Second, since the feature correspondence function is based on a state describing the relation of the single scans (e.g. the robots' poses), these algorithms are depending on 
sufficiently good state initialization to avoid local minima. In this paper, we suggest a solution to the first problem: correct alignment in the absence of sufficient feature correspondences. This problem can e.g. arise in search and rescue environments (these environments typically show a little number of landmarks only) or when multiple robots team to build a joint global map. In this situation, single scans, acquired from different views, do not necessarily reveal the entire structure of the scanned object. The motivation to our approach is that even if the optimal relation between single scans is not known, it is possible to infer hypotheses of underlying structures from the non-optimal combination of single scans based on the assumption of certain real world knowledge. Figure 1 illustrates the basic

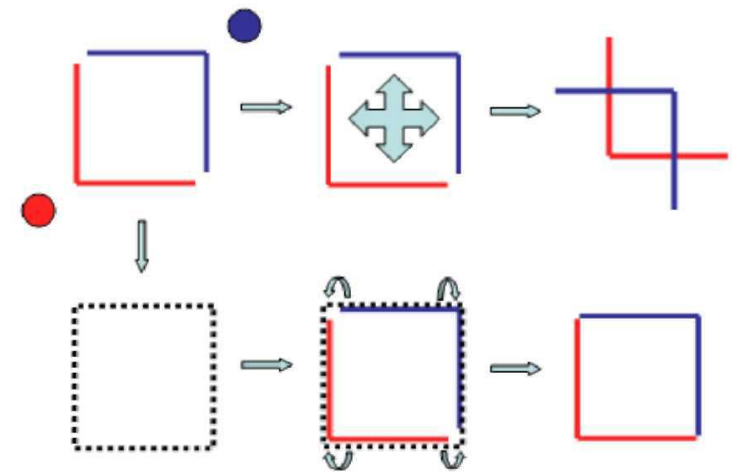

Fig. 1. Motivation of Virtual Scan approach (a-f in reading order): a) rectangular object scanned from two positions (red/blue robots). b) correspondence between single scans (red/blue) does not reveal the scanned structure c) misalignment due to wrong correspondences d) analysis of estimated global map detects structure e) structure is added as Virtual Scan f) correct alignment achieved due to correspondences between real world scans and Virtual Scans

idea. It shows a situation where the relation between features of single scans can not reveal the real world structure, and therefore leads to misalignment. Analysis from a global view estimates the underlying structure. This hypothesis then augments the real world data set, to achieve a correct result.

The motivational example shows the ideal case; it doesn't assume any error in the global map estimation (the relative pose between red and blue scan), hence it is trivial to detect the correct structure. Our system also handles the non ideal situation including pose errors. It utilizes a feedback structure between hypothesis generation and real data alignment response. The feedback iteratively adjusts the hypotheses to the real data (and vice versa). This will be discussed in more detail below. We first want to explain our approach in a more general framework.

Feature correspondence algorithms, e.g. in ICP or FFS, can be seen as low level spatial cognition processes (LLSC), since they operate based on low level geometric information. The feature analysis of the global map, which is suggested in this paper, can be described as mid level spatial cognition process (MLSC), since we aim at analysis of features like lines, rectangles, etc. Augmenting real world data with ideal models of expected data can be seen as an example of integration of LLSC and MLSC processes to improve the performance of 
spatial recognition tasks in robotics. We are using the area of robot perception for mobile rescue robots, specifically alignment of $2 \mathrm{D}$ laser scans, as a showcase to demonstrate the advantages of these processes.

In robot cognition, MLSC processes infer the presence of mid level features from low level data based on regional properties of the data. In our case, we detect the presence of simple mid level objects, i.e. line segments and rectangles. The MLSC processes model world knowledge, or assumptions about the environment. In our setting for search and rescue environments, we assume the presence of (collapsed) walls and other man made structures. If possible wall-like elements or elements somewhat resembling rectangular structures are detected, our system generates the most likely ideal model as a hypothesis, called 'Virtual Scan'. Virtual Scans are generated from the ideal, expected model in the same data format as the raw sensor data, hence Virtual Scans are added to the original scan data indistinguishably for the low level alignment process; the alignment is then performed on the augmented data set.

In robot cognition, LLSC processes usually describe feature extraction based on local properties like spatial proximity, e.g. based on metric inferences on data points, like edges in images or laser reflection points. In our system laser scans (virtual or real) are aligned to a global map using mainly features of local proximity using the LLSC core process of 'Force Field Simulation' (FFS). FFS was recently introduced to robotics [20]. In FFS, each data point can be assigned a weight, or value of certainty. It also does not make a hard, but soft decision about the data correspondences as a basis for the alignment. Both features make FFS a natural choice over its main competitor, ICP [2, 24], for the combination with Virtual Scans. The weight parameter can be utilized to indicate the strength of hypotheses, represented by the weight of virtual data.

FFS is an iterative alignment algorithm. The two levels (LLSC: data alignment by FFS, MLSC: data augmentation) are connected by a feedback structure, which is repeated in each iteration:

- The FFS-low-level-instances pre-process the data. They find correspondences based on low level features. The low level processing builds a current version of the global map, which assists the mid-level feature detection

- The mid level cognition module analyzes the current global map, detects possible mid level objects and models ideal hypothetical sources possibly

being present in the real world. These can be seen as suggestions, fed back into the low level system by Virtual Scans. The low level system in turn adjusts its processing for re-evaluation by the mid level systems. 


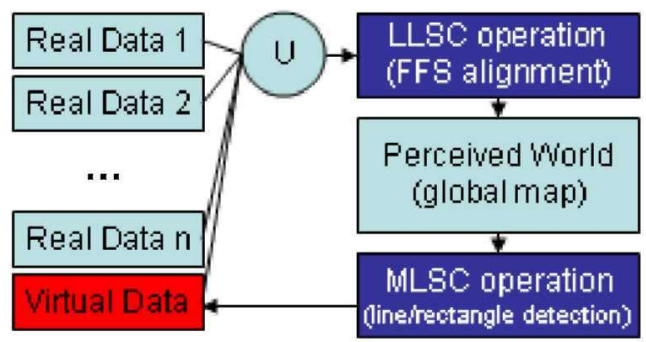

Fig. 2. LLSC/MLSC feedback. The LLSC module works on the union of real scans and the Virtual Scan. The MLSC module in turn re-creates a new Virtual Scan based on the result of the LLSC module.

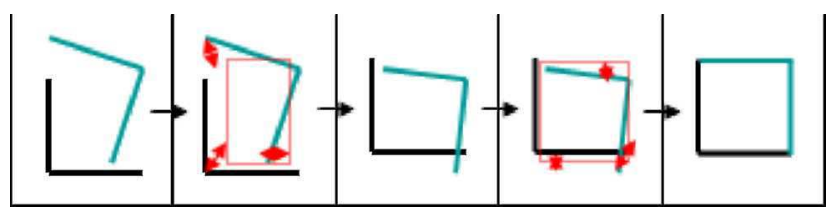

Fig. 3. Feedback between Virtual Scans (VS) and FFS. From left to right: a) Initial state of real data. b) Real data augmented by VS (red). c) After one iteration using real and virtual scans. d) new hypothesis (red) based on (c). e) next iteration. Since this results resembles an ideal rectangle, adding a VS would not relocate the scans. The system converged.

The following example will illustrate the feedback: Figure 3 assumes two scans, e.g. taken from robots in two different positions (compare to fig.1). An MLSC process detects a rectangular structure (the asumed world knowledge) and adds an optimal generating model to the data set. The LLSC module aligns the augmented data. The hypothesis now directs the scans to a better location. In each iteration, the relocated real scans are analyzed to adjust the MLSC hypothesis: LLSC and MLSC assist each other in a feedback loop.

\section{Related Work in Spatial Cognition and Robot Mapping}

The potential of MLSC has been largely unexplored in robotics, since recent research mainly addressed LLSC systems. They show an astonishing performance: especially advances in statistical inferences $[5,10,13]$ in connection with geometric modeling of human perception $[6,9,25]$ and the usage of laser range scanners contributed to a breakthrough in robot applications, with the most spectacular results achieved in the 2005 DARPA Grand Challenge where several autonomous vehicles were able to successfully complete the race [26]. But although the work on sophisticated statistical and geometrical models like extended Kalman Filters (EKF),e.g. [12], Particle Filters [10] and ICP (Iterative Closest Point) $[2,24]$ utilized in mapping approaches show impressive results, their limits are clearly visible, e.g. in the aforementioned rescue scenarios. These systems are still based on low level cognitive features, since they construct metric maps using correspondences between sensor data points. However, having these well-engineered low level systems at hand, it is natural to connect them to MLSC processes to mutually assist each other.

The knowledge in the area of MLSC in humans, in particular in spatial intelligence and learning, is advancing rapidly [7, 14, 27]. Research in AI models such results to generate 
generic representations of space for mobile robots using both symbolic, e.g. [16], and non symbolic, e.g. [8], approaches. Each is trying to identify various aspects of the cognitive mapping process. Naturally, SLAM (Simultaneous Localization and Mapping [4] is often used as an application example [23]. In [28], a spatial cognition based map is generated based on High Level Objects. Representation of space is mostly based on the notion of a hierarchical representation of space. Kuipers [16] suggests a general framework for a Spatial Semantic Hierarchy (SSH), which organizes spatial knowledge representations into levels according to ontology from sensory to metrical information. SSH is an attempt to understand and conceptualize the cognitive map [15], the way we believe humans understand space. More recently, Yeap and Jefferies [29] trace the theories of early cognitive mapping. They classify representations as being space-based and object-based. Comparing to our framework, these classifications could be described being related to LLSC and High Level Spatial Cognition (HLSC), hence the supposed LLSC/MLSC system would relate closer to space-based systems.

In [1], the importance of 'Mental Imagery' in (Spatial) Cognition is emphasized and basic requirements of modeling are stated. Mental Images invent or recreate experiences to resemble actually perceived events or objects. This is closely related to the "Virtual Scans" described in this proposal. Recently, Chang et al. [3] presented a predictive mapping approach (P-SLAM), which analyzes the environment for repetitive structures on the LLSC level (lines and corners) to generate a "virtual map". This map is either used as a hypothesis in unexplored regions to speed up the mapping process or as an initialization help for the utilized particle filters when a region is first explored. In the second case the approach has principles similar to the presented Virtual Scans. The impressive results of P-SLAM can also be seen as proof of concept of integrating prediction into robot perception.

The problem of geometric robot mapping is based on aligning a set of scans. On the LLSC level the problem of simultaneous aligning of scans has been treated as estimating sets of poses [22]. The underlying framework for such a technique is to optimize a constraintgraph, in which nodes are features, poses and edges are constraints built using various observations and measurements.

There are numerous image registration techniques, the most famous being Iterative Closest Point (ICP)[2], and its numerous variants to improve speed and converge basins. Basically all these techniques do search in transformation space trying to find the set of pair-wise transformations of scans by optimizing some function defined on transformation space. The techniques vary in defining the optimization functions that range from being error metrics like "sum of least square distances" to quality metrics like "image distance". 'Force Field Simulation' (FFS), [20], minimizes a potential derived from forces between corresponding data points. The Virtual Scan technique presented in this paper will interact with FFS as underlying alignment technique.

\section{Scan Alignment using Force Field Simulation}

The understanding of FFS is crucial to the understanding of the presented extension of the FFS alignment using Virtual Scans. We will give an overview here. FFS aligns single scans $S_{i}$ obtained by robots, typically from different positions. We assume the scans to be roughly pre-aligned (see fig.11), e.g. by odometry or shape based pre-alignment. This is in accord with the performance comparison between FFS and ICP described in [19]. FFS alignment, in 
detail described in [20] is able to iteratively refine such an alignment based on the scan data only. In FFS, each single scan is seen as a non-deformable entity, a 'rigid body'. In each iteration, a translation and rotation is computed for each single scan simultaneously. This process minimizes a target function, the 'point potential', which is defined on the set of all data points (real and Virtual Scans: FFS cannot distinguish). FFS solves the alignment problem as optimization problem utilizing a gradient descent approach motivated by simulation of dynamics of rigid bodies (the scans) in gravitational fields, but " replaces laws of physics with constraints derived from human perception" [20]. The gravitational field is based on a correspondence function between all pairs of data points, the 'force' function. FFS minimizes the overlaying potential function induced by the force and converges towards a local minimum of the potential, representing a locally optimal transformation of scans. The force function is designed in a manner that a low potential corresponds to a visually good appearance of the global map. As scans are moved according to the laws of motion of rigid bodies in a force field, single scans are not deformed.

Fig. 4 shows the basic principle: forces (red arrows) are computed between 4 single scans (the 4 corners). FFS simultaneously transforms all scans until a stable configuration is gained.

$$
V\left(v_{1}, v_{2}\right)=M\left(v_{1}, v_{2}\right) \frac{v_{i}-u_{j}}{\left\|v_{i}-u_{j}\right\|}
$$

Its magnitude $\backslash \backslash \mathrm{M}\left(v_{i}, U j\right) \|=C\left(v_{i}, U j\right)$ is defined as:

$$
M\left(v_{i}, u_{j}\right)=\frac{1}{\sigma_{t} \sqrt{2 \pi}} e^{\left(-\frac{\left\|v_{i}, u_{j}\right\|^{2}}{2 \sigma_{t}^{2}}\right)} w_{i} w_{j} \cos \left(\measuredangle\left(v_{i}, u_{j}\right)\right)
$$

With $\mathrm{Si}, \mathrm{S}_{2}$ being two different scans, the force between two single data points $\mathrm{v}_{\mathrm{i}} G S_{1}$ and $u j$ $G \mathrm{~S}_{2}$ is defined as a vector with parameters at, $W, W j, Z\left(\mathbf{v}_{\mathbf{i}}, \mathbf{u}_{\mathbf{j}^{-}}\right)$defined as follows: $Z\left(\mathbf{v}_{\mathbf{i}}, \mathbf{u}_{\mathbf{j}^{-}}\right)$ denotes the angle between the directions of points, which is defined as the angle between directions of assumed underlying locally linear structures. See fig. 5, left, for an example, which especially shows the influence of the cosine-term in eq.2: forces are strong between parallel structures only. In eq.2, the forces are strongly depending on at, which is a parameter steering the radius of influence. With at decreasing during the iterative process, FFS changes the influence of each data point from global to local. In addition, the weight $\mathbf{w}_{\mathbf{i}}, \mathbf{w}_{\mathbf{j}} \mathbf{-}$ (or mass) determines the influence of points $\mathbf{v}_{\mathbf{i}}, \mathbf{U} \mathbf{j}$. The weight is a parameter which can e.g. express the certainty about a point, or it can model the feature importance. We utilize this feature of FFS to model the strength of hypothesis in the Virtual Scans. Hence in eq. 2 the interfacing between LLSC and MLSC can be seen directly: distance and cosine term refer to LLSC, while the weights are derived from MLSC (in case of the Virtual Scans).

To compute the resulting movement from the forces of all point pairs between different scans, FFS re-assigns a constant mass to all data points and applies Newton's law of movement of rigid bodies in force fields. Constant mass causes data points participating in stronger force relations to influence the transformation stronger than those responding to weaker forces. For a single transformation step see fig. 5, right.

The step width $A_{t}$ of the gradient descent step in FFS is determinded by a 'cooling process'. $A_{t}$ It is monotonically decreasing, allowing the system in early iterations to jump out of local minima, yet to be attracted by local features in later steps. The interplay between $a_{t}$ and $A_{t}$ is an important feature of FFS. See figures 11 and 12 for an example of the performance of FFS on a laser range data set. 


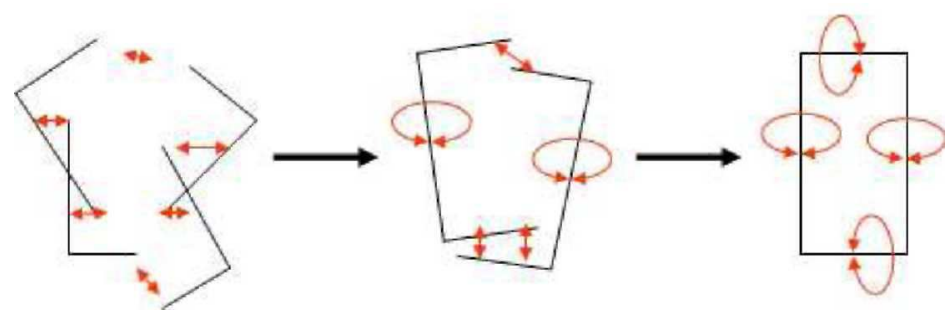

Fig. 4. Basic principle of FFS. Forces are computed between 4 single scans. Red arrows illustrate the principle of forces. The scans are iteratively (here: two iterations) transformed by translation and rotation until a stable configuration is achieved.

FFS is closely related to simultaneous ICP. A performance evaluation of both algorithms [19] showed similar results. In general, FFS can be seen as more robust with respect to global convergence with non near optimal initialization, since the point relations are not built in a hard (nearest neighbor) but soft(sum of forces) way. Also the inclusion of weight parameters makes it a natural decision for our purposes of extension using Virtual Scans.
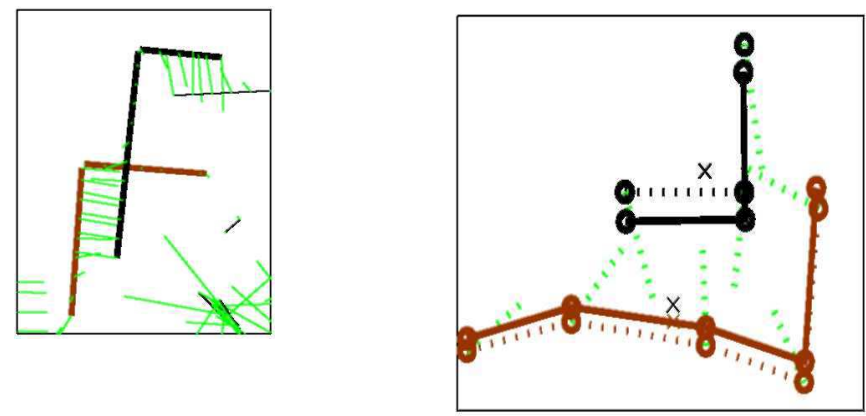

Fig. 5. FFS example. Left: Forces (green) between two rigid structures (brown, black). The black and brown lines connect the actual data set for display reasons only. The figure shows a magnification of the upper left corner of fig. 11, right. Right: example of force and movement. Dotted lines show 2 scans (black, brown) and their forces (green) in iteration $t$. Solid lines show the resulting transformed scans at iteration $t+1$.

\section{Creating Virtual Scans: Mid Level Analysis}

The point set used in our system is not the original raw data, but a re-sampled version; two pre-precessing steps are performed before the algorithm is applied. First, underlying linear structures (line segments) are detected in each single scan. Since line segments rely on local linearity of the underlying data points, classic global approaches like Hough line detection are not feasible. A recently published technique [21], using a statistical approach, 'Extended Expectation Maximization', is specifically tailored to model laser scan data with line segments. Second, having the line segments, new data points are generated in an equidistant way along these segments. The original data is discarded in favor of the newly generated 
points. This solves certain problems of FFS with unequally distributed sample point densities. It also reduces the number of points drastically. See fig. 6 for an example.

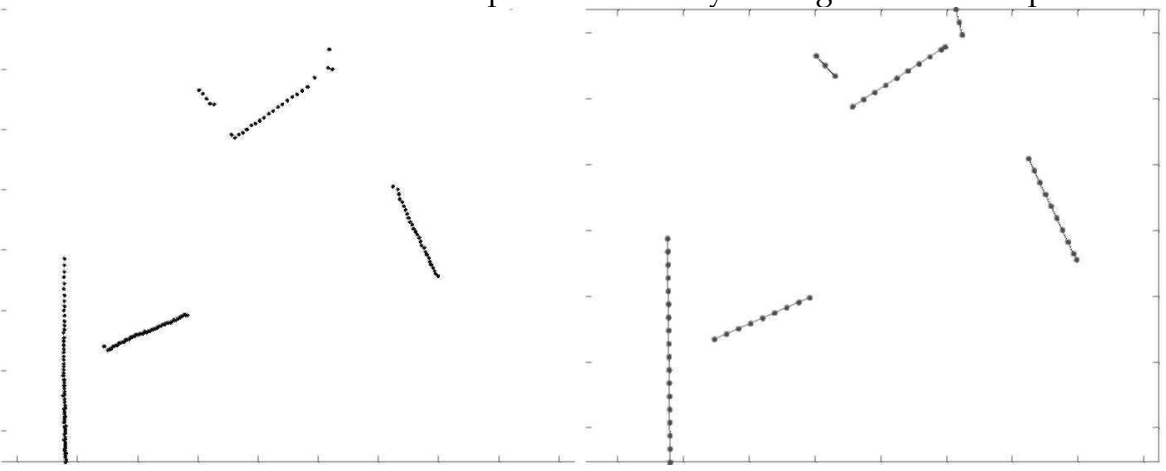

Fig. 6. FFS Pre-processing steps: left: original scan, right: line segments (black) and resampled point data (red)

The line and rectangle analysis is performed on the current, non augmented global map, i.e. the Virtual Scan of the previous iteration is discarded.

\subsection{Lines}

The usage of lines for our Virtual Scan approach is motivated by the world knowledge assumption of scanning a man made environment (e.g. a collapsed house): although these environments often locally don't show major linear elements any longer, a global view still often reveals an underlying global linear scheme, which we try to capture using a global line detection. Here (in contrast to our local line segment detection in the pre-processing step) we use the classic line detection approach of Hough transform [11], since it detects globally present linear structures. Hough transform does not only show location and direction of a line, but also the number of participating data points. We use this value to compute a certainty-of-presence measure, i.e. the strength of the line hypothesis. We only use lines above a certain threshold of certainty. We will specify below how the detected lines are utilized to create the Virtual Scan. The Hough detection is performed on the entire set of (resampled) data points. We do not use the local linear information of the line segment representation of the data here, since we aim at global linearity. This is more robustly detected by Hough transform.

\subsection{Rectangles}

The rectangle detection operates on the entire set of line segments of all scans, gained in preprocessing step 1. We use a rectangle detection approach described in [17]: each line segment (of each single scan) is translated into 'S,L,D space' (Slope,Length,Distance), which simplifies the detection of appropriate (rectangular like) configurations of four near parallel and near perpendicular segments. For details see [17]. Superimposing all line segments at an early stage of FFS leads to an additional problem, due to still imprecise pose estimation. Single lines in the environment, present in multiple single scans but not aligned perfectly yet, are represented by clusters of many segments, rather than the required single segment. We therefore merge similar lines in a cluster to a single prototype using a line merge 
approach described in [18], see figure 7. The rectangle detection module then predicts location, dimension and certainty-of-presence of hypothetical, ideal rectangles present in the data set of merged line segments. The certainty, or strength of the hypothesis is derived from properties (segment length, perpendicularity) of participating rectangle-generating line segments. This value is used to create the weight of the rectangle in the Virtual Scan.

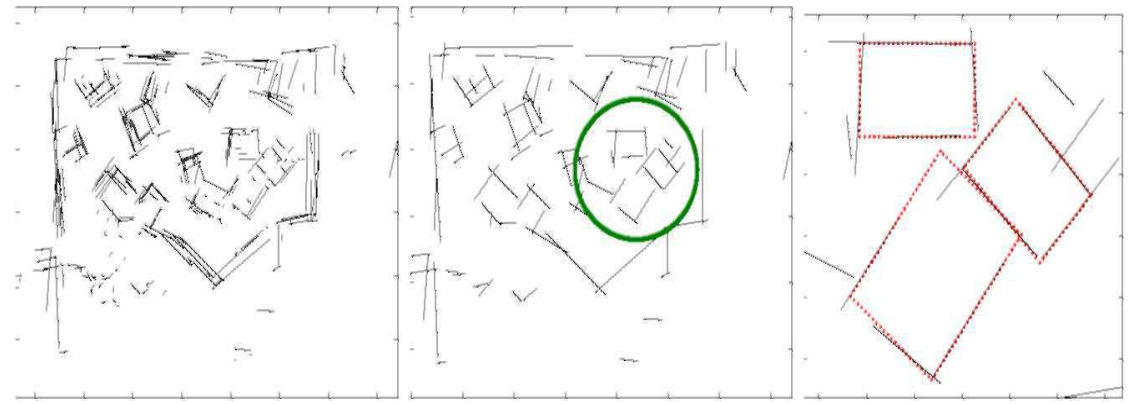

Fig. 7. Rectangle detection. a) Global map built by line segments of all single scans. b) result of global line merging c) red: detected rectangles (magnification of area encircled in b)

\subsection{Creating a Virtual Scan}

A Virtual Scan is a set of virtual laser scan points, superimposed over the entire area of the global map. The detected line segments and rectangles are 'plotted' into the Virtual Scans, i.e. they are represented by point sets as if they would be detected by a laser scanner. We assume a virtual laser scanner that represents each line and rectangle by a set of points, sub sampled equidistantly according to the point density of the underlying point data in the original data set. All detected elements (lines, rectangles) are plotted into a single Virtual Scan.

An important feature of the Virtual Scan is that each scan point is assigned a weight, representing the strength of hypothesis of the generating virtual structure. Utilizing this feature, we benefit from the weights that steer the FFS alignment. As defined in eq.2, the weight $W i, W j$ directly influences the alignment process; stronger points, i.e. points with higher value $w_{i}$, have a stronger attraction. Hence, a strong hypothesis translates into a locally strongly attractive structure. The hypothesis value reflects the belief into the hypothesis relative to the real data; all data points of the real data are assigned a 'normal' weight of 1 .

\section{Alignment using Virtual Scans: Algorithm}

The algorithm describes the interplay between LLSC (FFS) and the MLSC analysis. $S_{i}, \mathrm{i}=$ 1..n, denotes the real scan data, consisting of $\mathrm{n}$ scans. V[tt is the Virtual Scan in iteration $\mathrm{t}$. Init: $t=1, V t^{\circ}=0$, create set of line segments $L_{i}$ for each scan $S_{i}$

1) Perform FFS on $\left(\mathrm{J}_{i=1} n\right.$ Si $U V^{[t-1} \backslash$ resulting in transformations (translation, rotation) Ti ${ }^{[t]}$ for each scan $\mathrm{Si}=\mathrm{i}$..n

2) Form global map G of points and GL of line segments, superimposing the transformed scans and their line segment representation: $\mathrm{G}=\left(\mathrm{J}_{n} \mathrm{~T}^{\prime \prime} \mathrm{Jt}\right](\mathrm{Si}), \mathrm{GL}=\left(\mathrm{J}_{n} \mathrm{~T}^{\prime \prime} \mathrm{Jt}(\mathrm{Li})\right.$ 
3) Detect set of lines $L$ in $G$, set of rectangles $R$ in GL

4) Create Virtual Scan VM. VM contains scan points representing the elements of L and R.

5) Compute parameters $a t$ and At for the FFS process

6) Loop: goto 1, or end if FFS converged (stable global map).

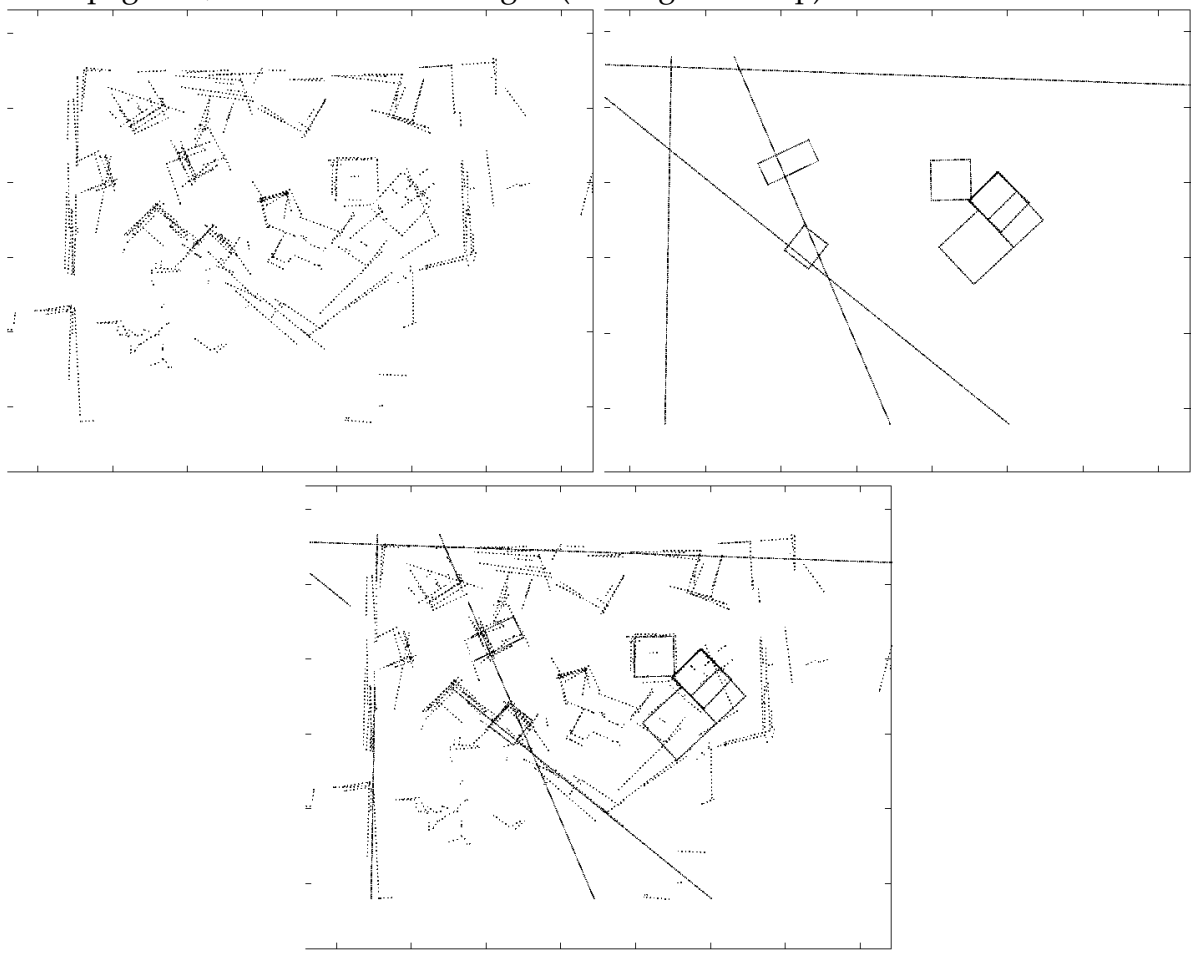

Fig. 8. Virtual Scans in an early stage of FFS. a) global map b) the Virtual Scan consisting of points representing detected lines and rectangles c) superimposition of real data and Virtual Scan. This is the data used in the next FFS iteration.

\section{Experiments and Results}

\subsection{Sparse Scanning (Simulated Data)}

This experiment shows the effect of Virtual Scans in a sparsely scanned environment. It features a simple environment to highlight the principle of Virtual Scans and to show the improvement in the alignment process. Please compare also to the motivational example in the introduction, as well as to figures 1 and 3. A simulated arena, consisting of 4 rectangular rooms, is scanned from 5 different positions. Each single scan is translated and rotated to simulate pose errors, and pre-processed see fig.9,a,b). We first try to align this data set using FFS without Virtual Scans. The performance of FFS depends on the initial value of at (see eq.2), at $=0$. at changes the radius of influence of neighboring points. We tried multiple initial 


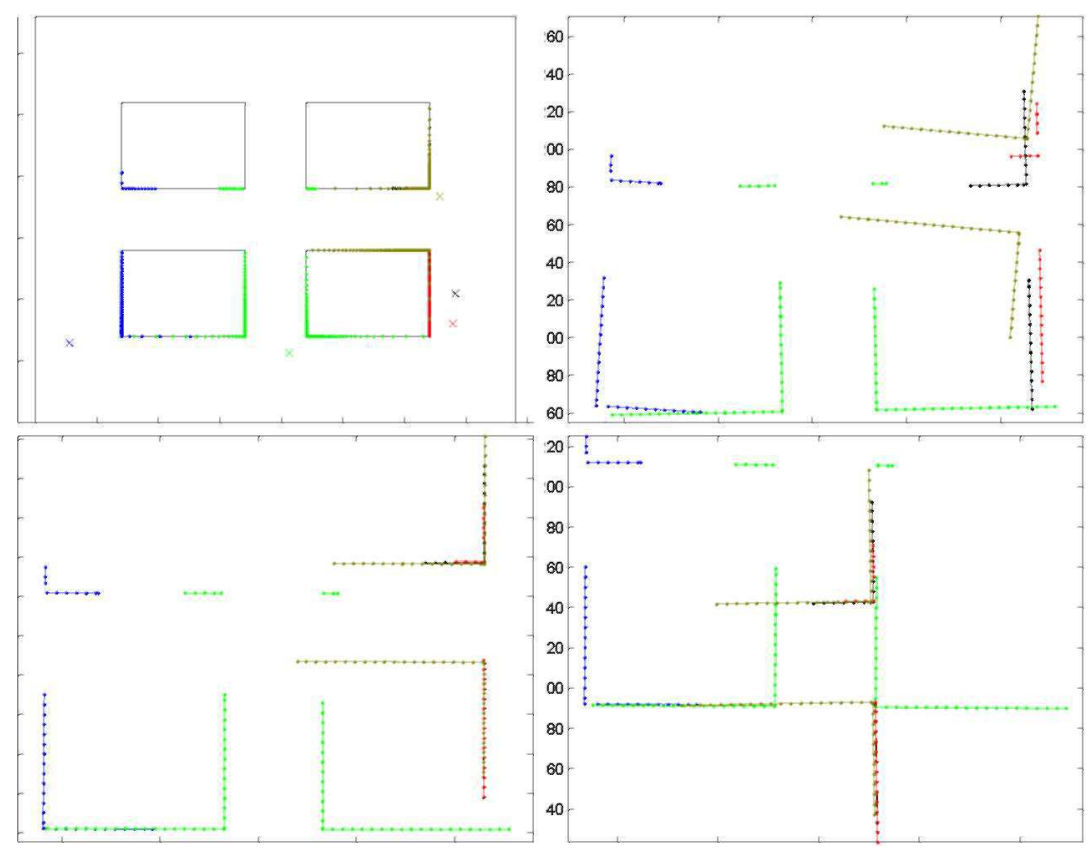

Fig. 9. (in reading order): a)simulated arena, scanned from 5 positions (crosses). Points of same color belong to same scan. b) After adding pose error to the data of (a) and preprocessing: underlying segments of (a) and re-sampled point data. c/d) result of FFS without Virtual Scans, intialized with configuration in (b). c) $<7 \mathrm{t}=\mathrm{O}=30 \mathrm{~d}$ ) $<7 \mathrm{t}=\mathrm{O}=80$

values, results of at $=0=30$ and at $=0=80$ are shown (a in units of the data set: the width of the simulated arena is 400 units), see fig.9c),d). In c), with a low at $=0$, local structures are captured and aligned correctly, but global correspondences can not be detected (the 'hallway' between the rooms shows an incorrect offset). Increasing $a_{t}={ }_{0}$ and therewith strengthening the influence of global structures in d) leads to wrong results since local correspondences become relatively unimportant: FFS optimizes correspondences of major structures (although they are distant from each other in the initial map). The disability of balancing the influence of local and global structures is is an inherent drawback of alignment processes which are based on point correspondences (e.g. ICP, FFS), and not a special flaw of FFS only (other values of $a$ did not improve the alignment).

Fig.10 shows the improvement using Virtual Scans. This experiment uses the same setting as experiment fig.9,c) $\left.\left(a_{t}=0=30\right)\right)$. FFS is able to detect correct local structures, and the global structures are captured through augmentation by Virtual Scans. Also the effect of the hypothesis adjustment by feedback is clearly visible: Fig.10a) shows an early hypothesis, which contains a wrong rectangle and misplaced lines. This early hypothesis is corrected by the feedback process between FFS and the rectangle/line detector. b)shows a later iteration, the line position is adjusted (though not perfect yet), 2 rectangle hypotheses compete (lower right corner). The final result is shown in c) and d). The detected lines adjusted expected 
global structures (the walls of the 'hallway') correctly, the winning rectangle hypothesis 'glued together' the corners of the bottom right room. Please notice that this room is a structure that is not entirely present in any single scan, but only detectable in the global map. Hence only the Virtual Scan enhanced FFS could perform correctly.

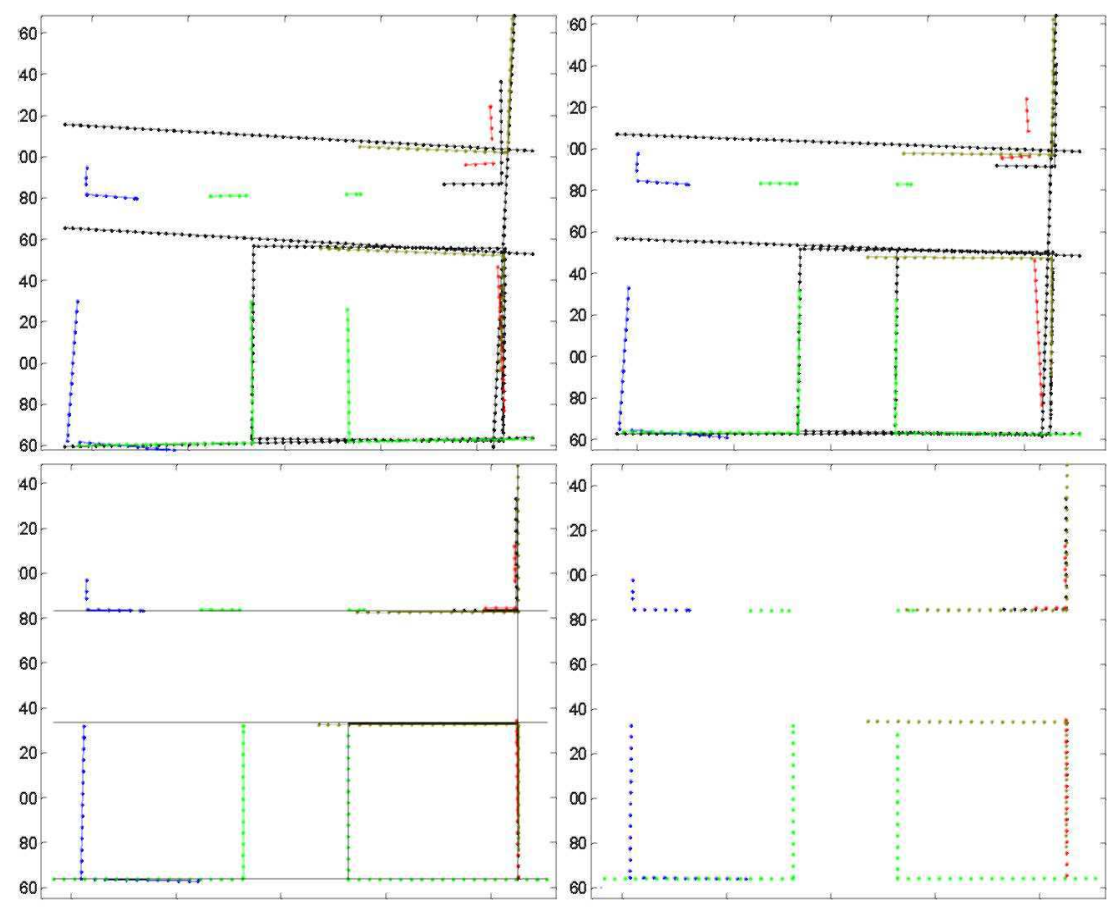

Fig. 10. Same experiment as in fig. 9, but using FFS $(<7 \mathrm{t}=\mathrm{o}=30)$ and Virtual Scans. Elements of Virtual Scans (lines and rectangles) are shown in black. In reading order: a) iteration 10, b) iteration $20 \mathrm{c}$ ) iteration 30 (final result) d) same as (c), but virtual scan not shown.

\subsection{NIST Disaster Area}

This experiment shows the improved performance of the alignment process on a real world data set. The data set consists of 60 single laser scans, taken from 15 different positions in 4 directions $(\mathrm{N}, \mathrm{W}, \mathrm{S}, \mathrm{E})$ with $20^{\circ}$ overlap. It can be interpreted as a scene scanned by 15 robots, 4 scans each. No order of scans is given. The scans resemble the situation of an indoors disaster scenery, scanned by multiple robots. The scans have little overlap and no distinct landmarks. The initial global map was computed using a shape based approach described in [19]. See fig. 11 for example scans and the initial map. We used the initial global map for two different runs of FFS, one using Virtual Scans, one without Virtual Scans. The increase in performance was evaluated by visual inspection, since for this data set no ground truth data is available. Comparing the final global maps of both runs, the utilization of Virtual Scans leads to distinct improvement in overall appearance and mapping details, see fig.12. 
Overall, the map is more 'straight' (compare e.g. the top wall), since the detection of globally present linear structures (top and left wall in fig.12) adjusts all participating single segments

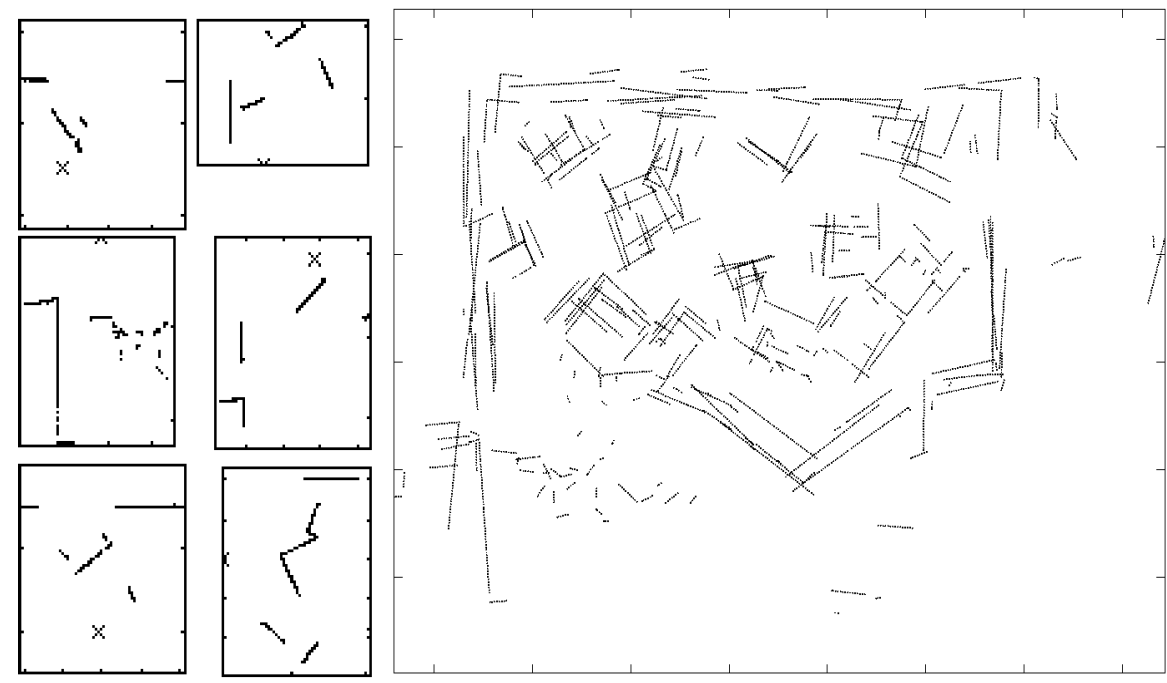

Fig. 11. The NIST disaster area data set. Left: 6 example scans (from a total of 60). Crosses show each robot's position. Right: 60 scans superimposed using a rough pre-estimation. This is the initial global map for the experiment in fig.12

to be collinear. These corrections advance into the entire structure.

The improvements can even better be seen in certain details, the most distinct ones encircled in fig.12, d),f). Especially the rectangle in the center of the global map is an excellent example for a situation where correct alignment is not achievable with low level knowledge only. Only the suggested rectangle from the Virtual Scan (see fig.12,c), can force the low level process to transform the scan correctly. Without the assumed rectangle the low level optimization process necessarily tried to superimpose 2 parallel sides of the rectangle to falsely appear as one (magnification of both situations in 12e).

\section{Conclusion and Outlook}

The presented implementation of an extension to the FFS alignment process using Virtual Scans containing hypothetical mid level real world structures could significantly improve the results for the alignment task. The implementation proves the applicability of the presented concept for the combination of LLSC and MLSC processes. The detection of simple elements (lines, rectangles) based on weak real world assumptions could improve the performance. We are aware that adding domain knowledge certainly enhances the risk of wrong inferences. The proposed system handles errors caused by premature belief in mid level features by implementing the feedback principle, which evaluates a single hypothesis. It is known that single hypothesis systems introducing higher knowledge tend to be not 
robust. Under certain circumstances this behavior could also be observed in experiments with our system, which needed manual parameter adjustment to steer the influence of the hypotheses. It can be embedded into a multiple hypotheses framework, e.g. particle filters, which will be part of future work.

Additional future work also has to determine the (geometric) level of elements which are meaningful enough to improve the alignment process, yet not too dominant. The current elements were chosen to model assumptions of indoor disaster areas. Future research about assumed real world elements will adjust to outdoor disaster settings.

\section{References}

[1] S. Bertel. Thomas barkowsky, dominik engel, christian freksa. computational modeling of reasoning with mental images: basic requirements. D. Fum, F. del Missier, A. Stocco (Eds.), Proceedings of the 7th International Conference on Cognitive Modeling ICCM06, 2006.

[2] P. Besl. N. mckay. a method for registration of 3.d shapes. IEEE PAMI, 14(2), 1992. [3] H. J. Chang. C.s.george lee and y.h. lu and y.c.hu. p-slam: Simultaneous localization and mapping with environmental structure prediction. IEEE Transactions on Robotics, Vol.23, No.2, April 2007.

[4] G. Dissanayake. H. durrant-whyte and t. bailey. a computationally efficient solution to the simultaneous localization and map building (slam) problem. ICRA2000 Workshop on Mobile Robot Navigation and Mapping, 2000.

[5] A. Doucet. N. defreitas, n. gordon. sequential monte carlo methods in practice. Springer, 2000.

[6] D. J. Field. A. hayes, r.f. hess. contour integration by the human visual system: evidence for a local association field. Vision Research, 33, pages 173-193, 1993.

[7] C. Freksa. M. knauff, b. krieg-brueckner, b. nebel, t. barkowsky. spatial cognition iv: Reasoning, action, interaction. Springer, 2004.

[8] T. Ghiselli-Crippa. Hirtle s.c., munro, p. connectionist models in spatial cognition. The Construction of Cognitive Maps, Kluwer Academic Publishers. 1-51, pages 87-104, 1996.

[9] W. Grimson. Object recognition by computer: The role of geometric constraints. Boston, MA: MIT Press, 1991.

[10] G. Grisetti. C. stachniss, burgard w. improving grid-based slam with rao-blackwellized particle filters by adaptive proposals and selective resampling. ICRA, 2005.

[11] P. V. C. Hough. Methods and means for recognizing complex patterns. US patent 3,069,654, 1962.

[12] S. Huang. G. dissanayake. convergence analysis for extended kalman filter based slam. IEEE International Conference on Robotics and Automation, 2006.

[13] D. C. Knill. W. richards. perception as bayesian inference. New York: Cambridge, 1996.

[14] G. J. M. Kruijff. H. zender, p. jensfelt, h. i. christensen. situated dialogue and spatial organization: What, where and why? Int. J. of Advanced Robotic Systems, 2007.

[15] B. J. Kuipers. The cognitive map: 'could it have been any other way?'. Spatial Orientation: Theory, Research, and Application. New York: Plenum Press, pages 345359, 1983. 
[16] B. J. Kuipers. The spatial semantic hierarchy. Artificial Intelligence 119, pages 191-233, 2000.

[17] D. Lagunovsky. Ablameyko s. fast line and rectangle detection by clustering and grouping. Proc. of CAIP'97, Kiel, Germany, 1997.

[18] R. Lakaemper. L. j. latecki, d. wolter. geometric robot mapping. Int. Conf. on Discrete Geometry for Computer Imagery (DGCI), 2005.

[19] R. Lakaemper. A.nuechter, n.adluru. performance of $6 \mathrm{~d}$ lum and ffs slam. Workshop on Performance Metrics and Intelligent Systems (PerMIS), Gaithersburg, MD, August 2007.

[20] R. Lakaemper. N.adluru, 1.j.latecki, r.madhavan. multi robot mapping using force field simulation. Journal of Field Robotics, Special Issue on Quantitative Performance Evaluation of Robotic and Intelligent Systems, 2007.

[21] L. J. Latecki. R. lakaemper. polygonal approximation of laser range data based on perceptual grouping and em. IEEE Int. Conf. on Robotics and Automation (ICRA), Orlando, Florida, May 2006.

[22] F. Lu. Milios, e. globally consistent range scan alignment for environment mapping. Auton. Robots, 4(4), pages 333-349, 1997.

[23] A. Martinelli. A. tapus, k.o. arras, r. siegwart. multi-resolution slam for real world navigation. Proceedings of the 11th International Symposium of Robotics Research, Siena, Italy, 2003.

[24] A. Nuechter. K. lingemann, j. hertzberg, h. surmann, k. pervoelz, m. hennig, k. r. tiruchinapalli, r. worst, t. christaller. mapping of rescue environments with kurt3d. Proceedings of the International Workshop on Safety, Security and Rescue Robotics (SSRR '05),Kobe, Japan, June 2005.

[25] A. Pentland. Perceptual organization and the representation of natural form. AI Journal, Vol. 28, No. 2, pages 1-38, 1986.

[26] e. a. Thrun. Stanley: The robot that won the darpa grand challenge. Journal of Field Robotics 23(9), pages 661-692, 2006.

[27] D. Uttal. Seeing the big picture: Map use and the development of spatial cognition. Developmental Science, 3, pages 247-286, 2000.

[28] S. Vasudevan. V. nguyen, r. siegwart. towards a cognitive probabilistic representation of space for mobile robots. Proceedings of the IEEE International Conference on Information Acquisition (ICIA), Shandong, China, pages 20-23, August 2006.

[29] W. K. Yeap. M.e. jefferies. on early cognitive mapping. Spatial Cognition and Computation 2(2), pages 85-116, 2001. 


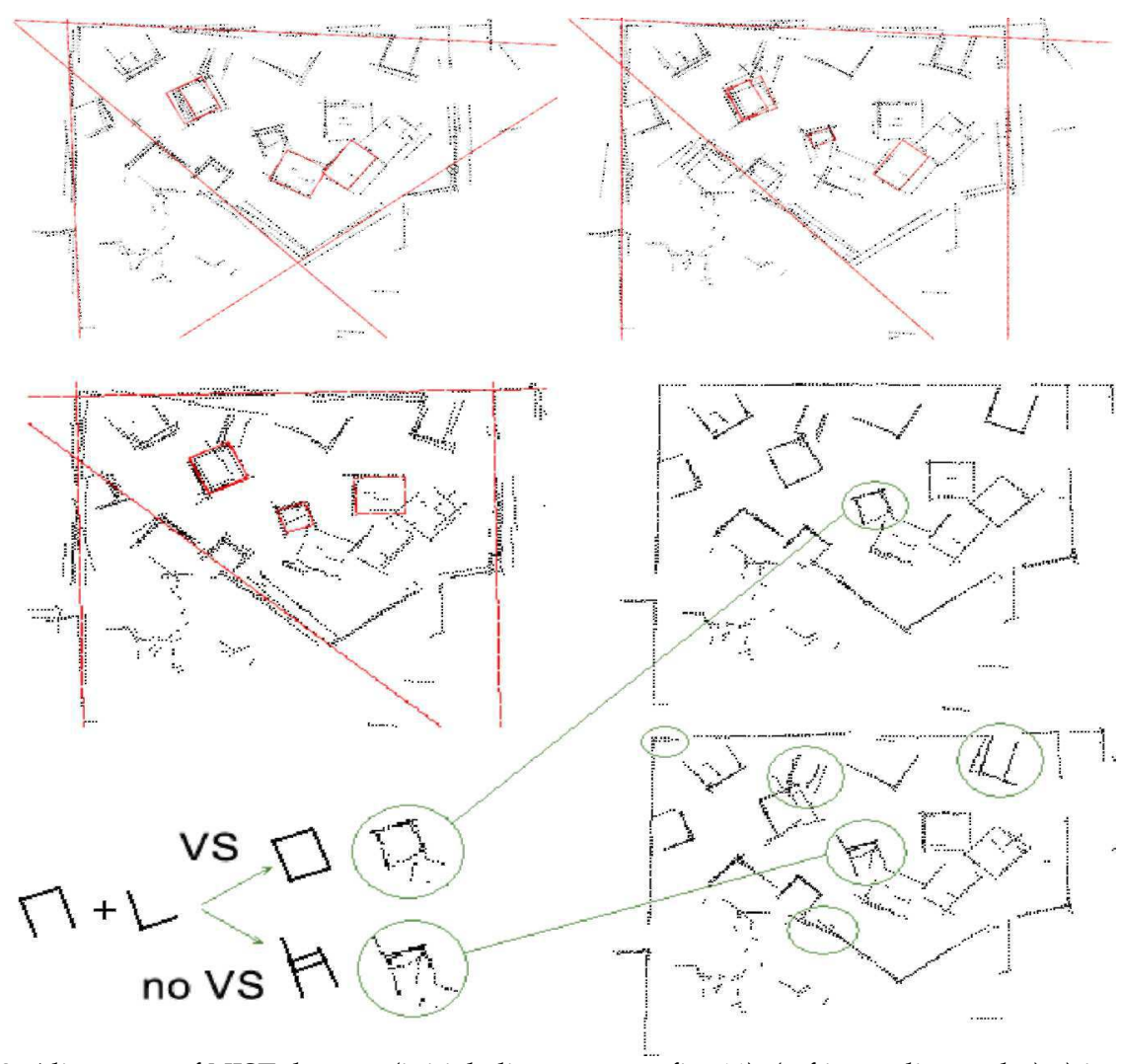

Fig. 12. Alignment of NIST data set (initial alignment see fig. 11). (a-f in reading order) a) iteration 4. b) iteration 8. c) iteration 10. In (a-c) red lines show the data of the Virtual Scan (VS). Please notice the mutual adjustment of hypotheses and real data. d) final result using Virtual Scans, after 100 iterations. The VS is not shown for clarity of display. Compare to f): final result of alignment without VS. Encircled areas show examples of improvement between (d) and (f). e) The center rectangle could only be aligned correctly using VS information. Please compare (e) to motivational examples fig. 1 and 3. 


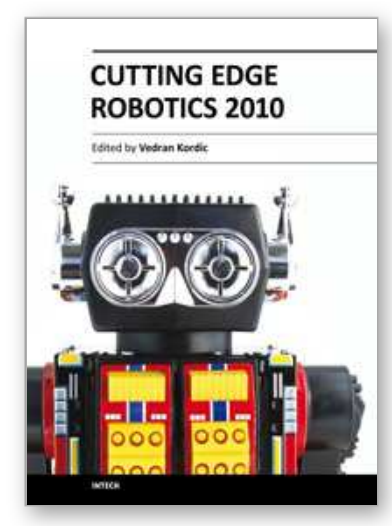

\author{
Cutting Edge Robotics 2010 \\ Edited by Vedran Kordic
}

ISBN 978-953-307-062-9

Hard cover, 440 pages

Publisher InTech

Published online 01, September, 2010

Published in print edition September, 2010

Robotics research, especially mobile robotics is a young field. Its roots include many engineering and scientific disciplines from mechanical, electrical and electronics engineering to computer, cognitive and social sciences. Each of this parent fields is exciting in its own way and has its share in different books. This book is a result of inspirations and contributions from many researchers worldwide. It presents a collection of a wide range of research results in robotics scientific community. We hope you will enjoy reading the book as much as we have enjoyed bringing it together for you.

\title{
How to reference
}

In order to correctly reference this scholarly work, feel free to copy and paste the following:

Rolf Lakaemper (2010). Augmenting Sparse Laser Scans with Virtual Scans to Improve the Performance of Alignment Algorithms, Cutting Edge Robotics 2010, Vedran Kordic (Ed.), ISBN: 978-953-307-062-9, InTech, Available from: http://www.intechopen.com/books/cutting-edge-robotics-2010/augmenting-sparse-laser-scanswith-virtual-scans-to-improve-the-performance-of-alignment-algorithms

\section{INTECH}

open science | open minds

\section{InTech Europe}

University Campus STeP Ri

Slavka Krautzeka 83/A

51000 Rijeka, Croatia

Phone: +385 (51) 770447

Fax: +385 (51) 686166

www.intechopen.com

\section{InTech China}

Unit 405, Office Block, Hotel Equatorial Shanghai

No.65, Yan An Road (West), Shanghai, 200040, China

中国上海市延安西路65号上海国际贵都大饭店办公楼405单元

Phone: +86-21-62489820

Fax: +86-21-62489821 
(C) 2010 The Author(s). Licensee IntechOpen. This chapter is distributed under the terms of the Creative Commons Attribution-NonCommercialShareAlike-3.0 License, which permits use, distribution and reproduction for non-commercial purposes, provided the original is properly cited and derivative works building on this content are distributed under the same license. 